\title{
THE REGULATION OF CORONAVIRUS GENE EXPRESSION
}

\author{
Stuart G. Siddell \\ Institute of Virology \\ University of Würzburg \\ Germany
}

\section{INTRODUCTION}

The coronaviruses are a monogeneric group of enveloped, RNA viruses that infect vertebrates, including man. In the main, coronavirus infections are associated with respiratory and gastrointestinal illness (Table 1, for a recent review of coronavirus biology see Spaan et al., 1990).

Table 1. Coronaviruses and their hosts

\begin{tabular}{|c|c|c|c|}
\hline & Acronym & Name & Host \\
\hline Group I & $\begin{array}{l}\text { MHV } \\
\text { HCVOC43 } \\
\text { HEV } \\
\text { BCV } \\
\text { TCV }\end{array}$ & $\begin{array}{l}\text { Murine hepatitis virus } \\
\text { Human coronavirus OC43 } \\
\text { Heamagglutinating encephalitis virus } \\
\text { Bovine coronavirus } \\
\text { Turkey coronavirus }\end{array}$ & $\begin{array}{l}\text { Mouse } \\
\text { Man } \\
\text { Pig } \\
\text { Cattle } \\
\text { Turkey }\end{array}$ \\
\hline Group II & $\begin{array}{l}\text { IBV } \\
\text { HCV229E } \\
\text { TGEV } \\
\text { CCV } \\
\text { FIPV }\end{array}$ & $\begin{array}{l}\text { Infectious bronchitis virus } \\
\text { Human coronavirus 229E } \\
\text { Transmissible gastroenteritis virus } \\
\text { Canine coronavirus } \\
\text { Feline infectious peritonitis virus }\end{array}$ & $\begin{array}{l}\text { Chicken } \\
\text { Man } \\
\text { Pig } \\
\text { Dog } \\
\text { Cat }\end{array}$ \\
\hline
\end{tabular}

In the last few years, our knowledge of coronavirus molecular biology has increased significantly. The basic principles of genomic organization and, at least, some aspects of the coronavirus replication strategy have been elucidated. Also, a number of mechanisms which regulate coronavirus gene expression at the translational level have been identified. This article briefly reviews this information. 


\section{GENOME ORGANIZATION}

The coronavirus genome is large, between 27 and 32 kilobases (kb) (Boursnell et al., 1987; Lee et al., 1991). At the present time, the complete genomic sequences of three coronaviruses (IBV, MHV and HCV229E) are available and partial, but quite extensive genomic sequences have been obtained for BCV, TGEV, CCV and FIPV. When this data is compared, it becomes apparent that the genetic organization of all coronaviruses is very similar but that two quite distinct lineages exist.

The overall genomic organization is illustrated in figure 1. The genes encoding the major structural proteins, $\mathbf{N}$ (nucleocapsid), $\mathbf{M}$ (membrane) and $\mathbf{S}$ (surface) are located towards the 3' end of the genome. These structural genes are interspersed by a number of open reading frames (ORFs) (e.g. IBV, 5; MHV, 3 and HCV229E, 3) which encode small (approx. 5-15 kilodalton, KDa) proteins of unknown function. The variable number and occurrence of deletion mutations in these ORFs (Yokomori and Lai, 1991; Wesley et al., 1990 ) suggests that they encode proteins that are not essential. One exception is the ORF located immediately upstream of the M protein gene (ORF 3c for IBV, ORF 4 for TGEV, etc) which encodes a protein that is incorporated into virions (Liu and Inglis, 1991; Godet et al., 1992). This protein displays striking structural similarities with the M2 protein of influenza virus.

Towards the 5 ' end of the genome are two large ORFs which overlap by 40-75 bases. These ORFs constitute the coronavirus RNA polymerase locus. The expression of gene products from this locus is complex and appears to involve mechanisms such as $(-1)$ ribosomal frameshifting and autoproteolytic processing of one or more polyproteins (Brierley et al., 1989; Denison and Perlman, 1986; Denison et al., 1991, 1992; Baker et al., 1989). The locus is polycistronic and 5 complementation groups of ts mutants with an RNA-minus phenotype have been mapped within this area by recombination analysis (Baric et al., 1990). The size of the locus (approximately $20 \mathrm{~kb}$ ) leads one to suspect that this region of the genome may also encode functions which are not related to RNA synthesis.

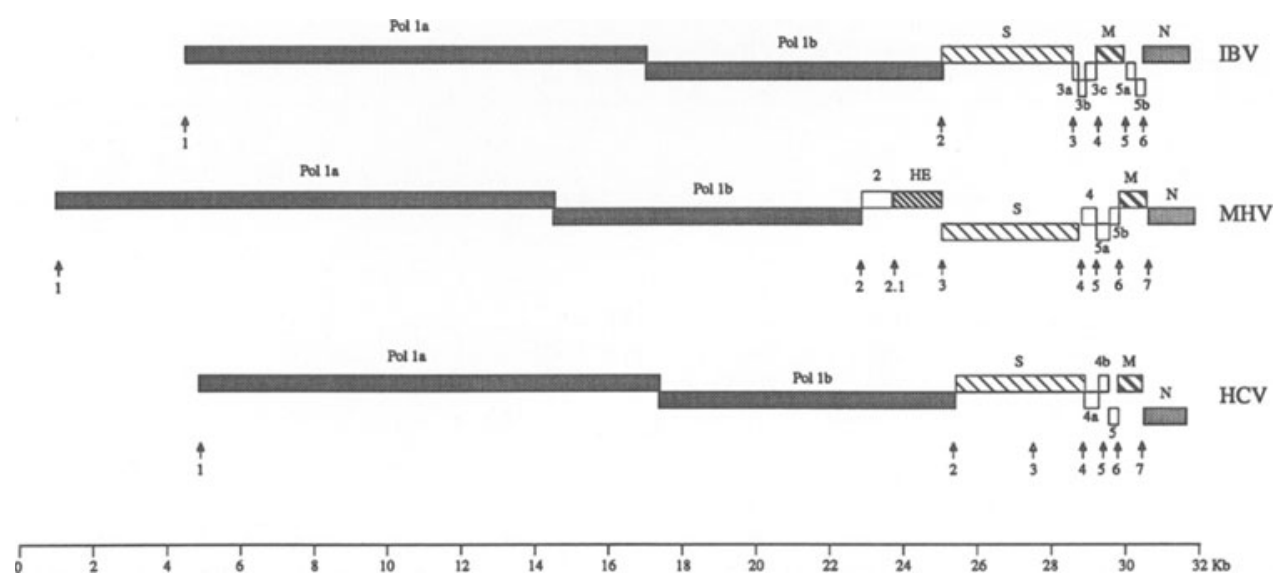

Figure 1. The organization of coronavirus genomes. The major open reading frames in the genomes of IBV, MHV and HCV229E are shown. The sizes of the ORFs are to scale.

Coronavirus genomes fall into two lineages: Group I, represented by MHV, HCVOC43, HEV, BCV and TCV and Group II, represented by IBV, HCV229E, TGEV, CCV and FIPV. These lineages manifest themselves as a clear distinction in the degree of genetic relatedness and the occurrence of an additional genetic element, comprised of two ORFs, ORF 2 and HE, which is found only in the Group I viruses (fig. 1). The HE gene encodes a fourth structural protein, the haemagglutinin-esterase, which shows a striking similarity with the HA1 region of the influenza C virus surface glycoprotein HEF (Luytjes et 
al., 1988) Spontaneous deletion mutants of MHV which do not express the HE protein or ORF 2 gene product (Luytjes et al., 1988, Schwarz et al., 1990) are still capable of efficient replication, at least in tissue culture.

\section{TRANSCRIPTION}

The expression of the coronavirus genome is mediated by genomic and subgenomic mRNAs. Together these mRNAs represent about $95 \%$ of the viral RNA present in the infected cell. $\mathrm{T}_{1}$ oligonucleotide analysis and sequencing of these mRNAs shows that they are structurally related as a $3^{\prime}$ coterminal (nested) set (fig. 2). Furthermore, at the $5^{\prime}$ end of each mRNA, a common sequence at about 70 nucleotides, the leader RNA, is present. This sequence is found only once in the genomic RNA, at the $5^{\prime}$ end, and the synthesis of the subgenomic mRNAs must, therefore, involve a process of discontinuous transcription. A specific motif, UCUAAAC, is found at those positions in the genome which define the 5' end of the unique region at each mRNA, i.e. the region not found in the next smallest RNA. This motif is thought to have an important function in mRNA synthesis, however, its precise role is still uncertain (for a review of coronavirus mRNA structure see Lai, 1990).

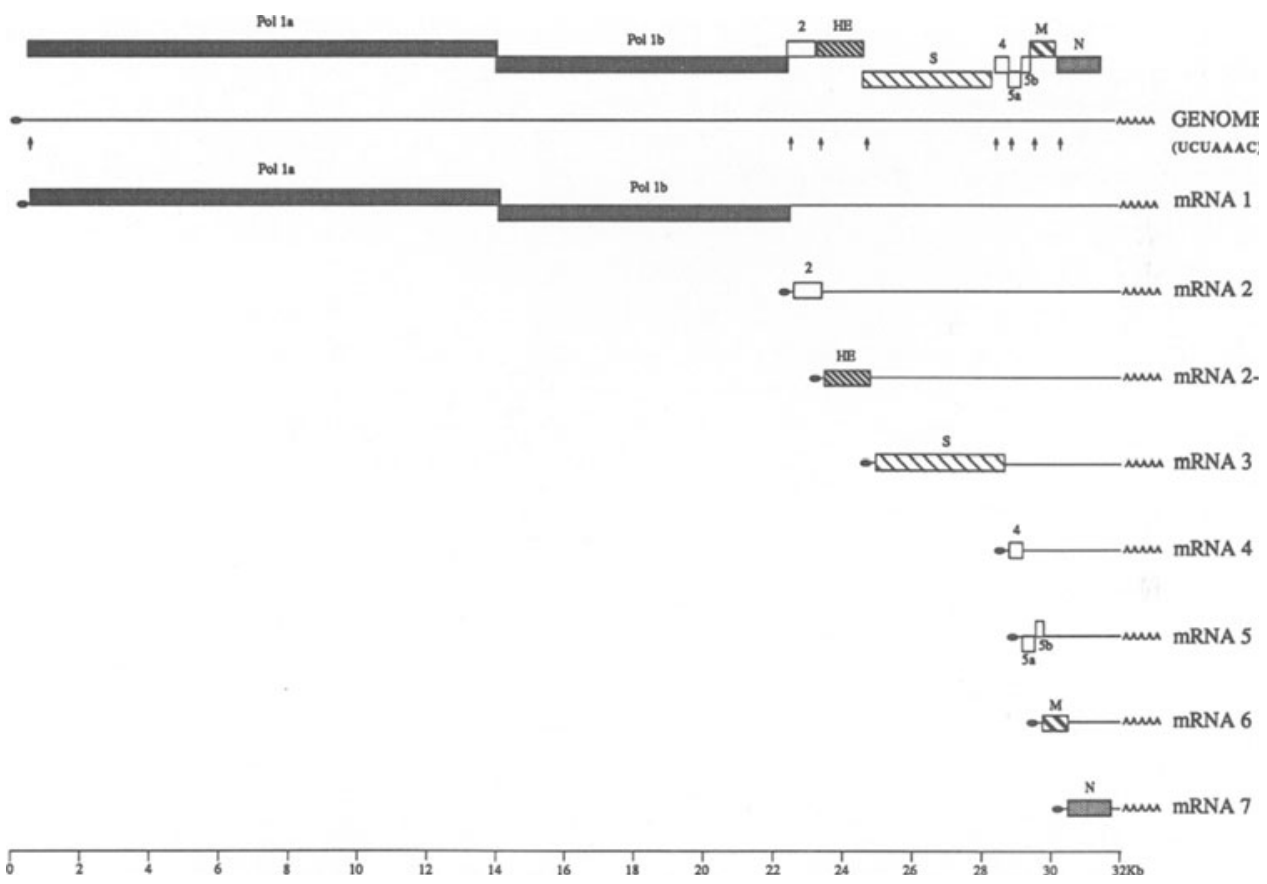

Figure 2. Expression of the coronavirus (MHV) genome. The arrangement of ORFs in the genome, their relationship to the $5^{\prime}$ unique regions of the subgenomic mRNAs and the positions of the UCUAAAC and leader RNA sequences in the genomic RNA are shown.

At the present time, it is not possible to present a definitive model for the genesis of coronavirus mRNAs. The following information is, however, relevant;

1. The dosage of UV-irradiation required to block the synthesis of the subgenomic mRNAs is proportional to their individual lengths (Jacobs et al., 1981, Stern and Sefton, 1982).

2. Infected cells contain subgenomic length negative strand RNAs which correspond to each of the subgenomic mRNAs (Sethna et al., 1989, Hofmann et al., 1990).

3. RNAse treatment of infected cell RNA yields one genomic and several subgenomic RF structures, the number of which corresponds to the number of subgenomic mRNAs (Hofmann et al., 1990, Sawicki and Sawicki, 1990). 
4. The sizes of the RF structures are proportional to the expected sizes of the negativestrand templates for each of the mRNA species (Sawicki and Sawicki, 1990).

5. The subgenomic RF structures are derived from transcriptionally active subgenomic RI structures (Sawicki and Sawicki 1990).

6. The RI structures synthesize both positive and negative strand RNA. The genomic and subgenomic RF structures and mRNAs are labelled coincidentally throughout this infection (Sawicki and Sawicki, personal communication).

7. The subgenomic negative-strand RNAs have a $3^{\prime}$ antileader sequence (Sethna et al., 1991).

With this information, at least, two quite different models for the genesis of coronavirus mRNA can be proposed. Both models assume that the genomic RNA is infectious (see Jeong and Makino, 1992) and both models propose that a process of "leaderprimed" discontinuous transcription, (Spaan et al., 1983, Baric et al., 1983) is used to generate, at least the initial subgenomic mRNA molecules. However, they differ from each other in the role postulated for the UCUAAAC motif. In the first model, which is termed "internal initiation" in figure 3 , this motif is an element which regulates the frequency of leader-primed initiation on genome length negative strand templates. Lai and coworkers (see Lai et al., 1990) originally proposed that this regulation was achieved by the degree of complementarity between the 3 ' end of the leader RNA and template sequences flanking the UCUAAAC motif. Later, Konings et al. (1988) argued that additional mechanisms had to be considered, for example, the role of transcriptional blockage at the initiation site. In any case, by analogy to other positive-strand RNA viruses, the model predicts subgenomic RFs corresponding to the unique regions of each mRNA and these structures have not yet been found (see Sawicki and Sawicki, 1990)
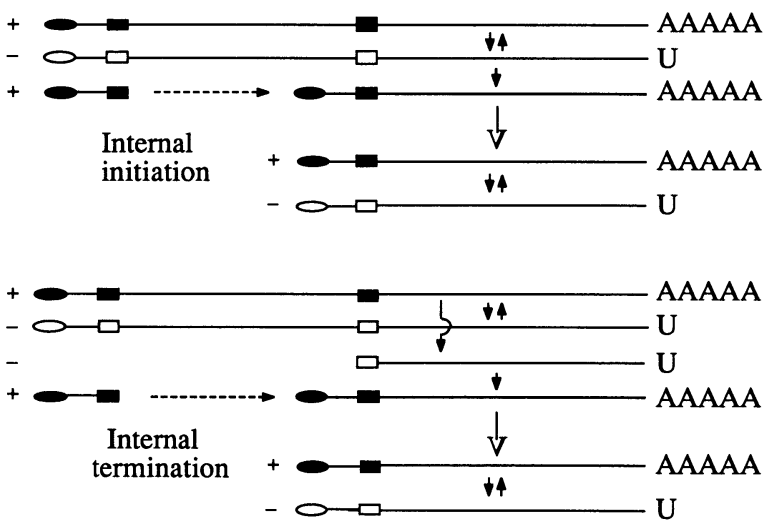

Figure 3. Two models for the genesis of coronavirus subgenomic mRNA.The leader RNA and UCUAAAC motifs are represented as oval and box structures respectively, in plus (closed) and minus (open) orientation. The molecules are not drawn to scale and only one subgenomic RNA is illustrated.

In the second model, termed "internal termination" in fig. 3, the role of the UCUAAAC motif is postulated to be a termination signal for negative strand RNA synthesis (Sawicki and Sawicki, 1990). In this way a (nested) set of $5^{\prime}$ coterminal negative strand RNAs are generated and these serve as templates for "leader-primed" transcription to produce subgenomic mRNA. It is possible that the UCUAAAC motif may also have a role in this event. In both models the subgenomic mRNAs can subsequently be copied to subgenomic negative-strand templates which possess antileader RNA, and subgenomic replicons are amplified independently of RNA synthesis involving genomic sized templates. The internal 
termination model is compatible with all of the available data to date, but important predictions, for example the presence of subgenomic negative-strand RNA lacking antileader sequences have not yet been fulfilled. Clearly, because of the exceedingly low amounts of viral RNA in the earliest stages of infection, it will be very difficult to distinguish the two models. Most probably, PCR technology and reverse genetics will be needed.

\section{TRANSLATION}

The basic premise of coronavirus translation is that only the information encoded in the 5' unique region of each mRNA is expressed from that RNA. This conclusion is based upon the in vitro translation of mRNAs (Rottier et al., 1981; Leibowitz et al., 1982, Siddell, 1983) and is consistent with the general requirements of translation in eucaryotic cells. For the majority of coronavirus mRNAs the 5' unique region encompasses only a single ORF. Thus, for example, the mRNAs encoding the structural proteins $\mathrm{N}, \mathrm{M}, \mathrm{S}$ and, if present, $\mathrm{HE}$ are functionally monocistronic. For some mRNAs, however, the 5 ' unique region contains more than one ORF. In these cases, additional strategies are used to regulate gene expression.

\section{Expression of the RNA Polymerase Locus (mRNA 1)}

As has been mentioned, the coronavirus RNA polymerase locus is polycistronic and is comprised of two large, overlapping ORFs, ORF 1a and ORF 1b. There is now substantial evidence that ORF $b$ is expressed by a (-1) ribosomal frame-shifting event mediated by a sequence specific element, the "slip-sequence" and an RNA structure, the RNA pseudoknot, both located at the ORF 1a/ORF $1 \mathrm{~b}$ junction (Brierley et al., 1987, Brierley et al., 1989, Bredenbeek et al., 1990). In the absence of proteolytic processing the polymerase locus could, therefore, encode a $400 \mathrm{KDa}$ OFR 1a product or a $700 \mathrm{KDa}$ ORF 1a-ORF $1 \mathrm{~b}$ product. Brierley and his colleagues have performed a detailed mutational analysis of the pseudoknot component of the IBV frameshifting signal (Brierley et al., 1991) and conclude that the efficiency of frameshifting, at least in vitro, can approach $30 \%$. Because of the small amounts of polymerase gene products synthesized in infected cells, it has not yet been possible to provide direct evidence for coronavirus ribosomal frame shifting in vivo.

The second level at which the expression of the coronavirus polymerase locus is regulated is proteolytic processing. Structural analysis of the IBV, MHV and HCV229E polymerase region has revealed sequence motifs characteristic of papain-like proteases and the poliovirus 3c-like protease (Boursnell et al., 1987, Lee et al., 1991, Herold and Siddell, unpublished). These "protease domains" are located in ORF 1a and an autoproteolytic activity has been mapped to the middle of this ORF by in vitro transcription/translation (Baker et al., 1989). Recently, the first steps in the analysis of proteolytic processing in relation to polymerase gene expression in vivo have been reported (Denison et al., 1991, 1992), but it is too early to propose a processing pathway.

\section{Functionally Polycistronic Subgenomic mRNAs}

Whereas most of the coronavirus subgenomic mRNAs contain only a single ORF in their 5' unique region, there is inevitabley at least one mRNA with two or three ORFs at this position. The best characterized examples are the mRNA 3 of IBV and the mRNA 5 of MHV. The IBV mRNA 3 has three ORFs in its 5' unique region and in infected cells all three translation products, 3a, 3b and 3c are expressed (Liu et al., 1991). The MHV mRNA 5 has two ORFs, $5 \mathrm{a}$ and $5 \mathrm{~b}$, and the downstream ORF product has been found in vivo (Leibowitz et al., 1988). A striking structural feature of both mRNAs is that the upstream ORFs (3a and 3b in IBV mRNA 3 and 5a in MHV mRNA 5) are, with the exception of the initiation codon, devoid of AUG triplets in any reading frame. This lead to the idea that expression of the downstream ORFs might be mediated by initiation events involving leaky scanning (Skinner et al., 1985, Budzilowicz and Weiss, 1987). This idea was, however, never confirmed experimentally and very recently Inglis and coworkers (Liu and Inglis, personal communication) have provided evidence that the IBV $3 c$ protein may be expressed by a mechanism involving the internal entry of ribosomes in a cap-independent fashion. 


\section{CONCLUSIONS}

The regulation of coronavirus gene expression appears to be complex. At the transcriptional level, coronavirus mRNAs are synthesized in a tightly regulated, nonequimolar fashion throughout the infection cycle. However, we do not yet have a clear picture of the mechanism of mRNA synthesis nor the regulatory controls which are used. At the translational level, coronaviruses appear to exploit a remarkable variety of strategies, including ribosomal frame-shifting, proteolytic processing and functionally polycistronic subgenomic mRNAs. The size and complexity of the coronavirus genome suggests that additional regulatory mechanisms have yet to be discovered.

\section{Acknowledgments}

I thank Drs S.G.and D.L. Sawicki, I. Brierley and S.C. Inglis for communicating results before publication. The authors work is supported by the DFG and the BMFT.

\section{References}

Baker, S.C., Shieh, C.-K., Soe, L.H., Chang, M.-F., Vannier, D.M., and Lai, M.M.C., 1989, Identification of a domain required for autoproteolytic cleavage of murine coronavirus gene A polyprotein, J. Virol. 63, 3693.

Baric, R.S., Fu, K.S., Schaad, M.C., and Stohlman, S.A., 1990, Establishing a genetic recombination map for MHV-A59 complementation groups, Virology 177:646.

Baric, R.S., Stohlman, S.A., and Lai, M.M.C., 1983, Characterization of replicative intermediate RNA of mouse hepatitis virus: presence of leader RNA sequences on nascent chains, $J$. Virol. 48:633.

Boursnell, M.E.G., Brown, T.D.K., Foulds, I.J., P.F., Tomley, F.M., and Binns, M.M., 1987, Completion of the sequence of the genome of the coronavirus avian infections bronchitis virus, J. Gen. Virol. 68:57.

Bredenbeek, P.J., Pachuk, C.J., Noten, A.F.H., Charité, A., Luytjes, W., Weiss, S.R., and Spaan, W.J.M., 1990, The primary structure and expression of the second open reading frame of the polymerase gene of the coronavirus MHV-A59; a highly conserved polymerase is expressed by an efficient ribosomal frame-shifting mechanism, Nucl. Acids Res. 18:1825.

Brierley, I., Boursnell, M.E.G., Binns, M.M., Bilimosia, B., Blok, V.C., Brown, T.D.K., and Inglis, S.C., 1987, An efficient ribosomal frame-shifting signal in the polymerase encoding region of the coronavirus IBV, EMBO J. 6:3779.

Brierley, I., Digard, P., and Inglis, S.C., 1989, Characterization of an efficient coronavirus ribosomal frameshifting signal: requirement for an RNA pseudoknot, Cell 57:537.

Brierley, I., Rolley, N.J., Jenner, A.J., and Inglis, S.C., 1991, Mutational analysis of the RNA pseudoknot component of a coronavirus ribosomal frame-shifting signal, J. Mol. Biol. 220:889.

Budzilowicz, C.J., and Weiss, S.R., 1987, In vitro synthesis of two polypeptides from a nonstructural gene of coronavirus mouse hepatitis virus strain A59, Virology 157:509.

Denison, M.R., and Perlman, S., 1986, Translation and processing of mouse hepatitis virus virion RNA in a cell-free system, J. Virol. 60:12.

Denison, M.R., Zoltick, P.W., Hughes, S.A., Giangreco, B., Olson, A.L., Perlman, S., Leibowitz, J.L., and Weiss, S.R., 1992, Intracellular processing of the N-terminal ORF 1a proteins of the coronavirus MHV-A59 requires multiple proteolytic events, Virology 189:274.

Denison, M.R., Zoltick, P.W., Leibowitz, J.L., Pachuk., C.J., and Weiss, S.R., 1991, Identifiction of polypeptides encoded in open reading frame $1 \mathrm{~b}$ of the putative polymerase gene of the murine coronavirus mouse hepatitis virus A59, J. Virol 65:3076.

Godet, M., L'Haridon, R., Vautherot, J.-F., and Laude, H., 1992, TGEV corona virus ORF 4 encodes a membrane protein that is incorporated into virions, Virology 188:666-675.

Hofmann, M.A., Sethna, P.B., and Brian, D.A., 1990, Bovine coronavirus mRNA replication continues throughout persistent infection in cell culture, J. Virol. 64:4108.

Jacobs, L., Spaan, W.J.M., Horzinek, M.C., and van der Zeijst, B.A.M., 1981, Synthesis of subgenomic mRNAs of mouse hepatitis virus is initiated independently: evidence from UV transcription mapping. J. Virol. 39:401.

.Jeong, Y.S., and Makino, S., 1992, Mechanism of coronavirus transcription: duration of primary transcription initiation activity and effects of subgenomic RNA transcription on RNA replication, J. Virol. 66, 3339.

Konings, D.A.M., Bredenbeek, P.J., Noten, J.F.H., Hogeweg, P., and Spaan, W.J.M., 1988, Differential premature termination of transcription as a proposed mechanism for the regulation of coronavirus gene expression, Nucl. Acids Res. 16:10849. 
Lai, M.M.C., 1990, Coronavirus: organization, replication and expression of genome, Ann. Rev. Microbiol. 44:303.

Lee, H.-J., Shieh, C.-K., Gorbalenya, A.E., Koonin, E.V., La Monica, N., Tuler, J., Bagdzhadzhyan, A., and Lai, M.M.C., 1991, The complete sequence (22 kilobases) of murine coronavirus gene encoding the putative proteases and RNA polymerase, Virology 180:567.

Leibowitz, J.L., Perlman, S., Weinstock, G., DeVries, J.R., Budzilowicz, C., Weissemann, J.M., and Weiss, S.R., 1988, Detection of a murine coronavirus non-structural protein encoded in a downstream open reading frame, Virology 164:156.

Leibowitz, J.L., Weiss, S.R., Paavola, E., and Bond, C.W., 1982, Cell free translation of murine coronavirus RNA, J. Virol. 43:905.

Liu, D.X., Cavanagh, D., Green, P., and Inglis, S.C., 1991, A polycistronic mRNA specified by the coronavirus infectious bronchitis virus, Virology 184:531.

Liu, D.X., and Inglis, S.C., 1991, Association of the infectious bronchitis virus 3c protein with the virion envelope, Virology 185:911.

Luytjes, W., Bredenbeek, P.J., Noten, A.F.H., Horzinek, M.C., and Spaan, W.J.M., 1988, Sequence of mouse hepatitis A59 mRNA 2: indications for RNA recombination between coronaviruses and influenza $C$ virus." Virology 166:415.

Rottier, P.J., Spaan, W.J.M., Horzinek, M.C., and van der Zeijst, B.A.M., 1981, Translation of three mouse hepatitis virus strain A59 subgenomic RNAs in Xenopus laevis oocytes, J. Virol. 38:20.

Sawicki, S.G., and Sawicki, D.L., 1990, Coronavirus transcription: subgenomic mouse hepatitis virus replicative intermediates function in RNA synthesis, J. Virol. 64:1050.

Schwarz, B., Routledge, E., and Siddell, S.G., 1990, Murine coronavirus non-structural protein ns2 is not essential for virus replication in transformed cells, J. Virol. 64:4784.

Sethna, P.B., Hofmann, M.A., and Brian, D.A., 1991, Minus-strand copies of replicating coronavirus mRNAs contain antileaders, J. Virol. 65:320.

Sethna, P.B., Hung, S.-L., and Brian, D.A., 1989, Coronavirus subgenomic minus-strand RNAs and the potential for mRNA replicons, Proc. Natl. Acad. Sci. USA 86:5626.

Siddell, S., 1983, Coronavirus JHM: coding assignments of subgenomic mRNAs, J. Gen. Virol. 64:113.

Skinner, M.A., Ebner, D., and Siddell, S.G., 1985, Coronavirus MHV-JHM mRNA 5 has a sequence arrangement which potentially allows translation of a second, downstream open reading frame. J. Gen. Virol. 66:581.

Spaan, W., Cavanagh, D., and Horzinek, M.C., 1990, Coronavirus, in "Immunochemistry of Viruses. II. The Basis for Serodiagnosis and Vaccines," M.H.V. van Regenmortel and A.R. Neurath, eds., Elsevier, Amsterdam.

Stern, D.F., and Sefton, B.M., 1982, Synthesis of coronavirus mRNAs: kinetics of inactivation of IBV RNA synthesis by UV light, J. Virol. 42:755.

Wesley, R.D., Woods, R.D., and Cheung, 1990, Genetic basis for the pathogenesis of transmissible gastroenteritis virus, J. Virol. 64:4761. 